\title{
Il costo medio per tipologia di prestazione nei centri trasfusionali
}

\author{
Silvia Martina*, Dante Cornago*, Livio Garattini*, Emanuela Castelnuovo*
}

\begin{abstract}
In Italy the collection, validation and separation of blood and its derivatives for transfusional interventions are carried out by some 380 transfusional centers (TCs), mainly located within greater health care structures. The aim of the CORSA (Costi Raccolta Sangue) study was to furnish an estimate of the mean cost of the main transfusional services on a sample of the Italian TCs. Of the centers contacted, only 13,3\% were able to deliver the unit costs necessary to perform the full-cost, fully-allocated analysis conducted. Of the three services examined (apheresis of plasma and platelets, whole blood collection), the selective collection of thrombocytes resulted the most expensive, as opposed to whole blood collection. The mean cost of a erythrocyte, plasma and platelet unit resulted of $17,7,21,6$ and $89,8 \cdot$, respectively, although the variation among centers was substantial.

To main conclusions can be drawn by the CORSA study. First, the very low percentage of TCs able to provide the necessary data for a complete economical analysis highlights an important delay in the modernizing process of health care management, started in 1992 with the aim to ameliorate resource allocation. Second, the study was not able to establish a relationship between production volume and production cost, indicating the absence of scale economy features in transfusional processes.
\end{abstract}

Farmeconomia e percorsi terapeutici 2003; 4 (1): 51-56

\section{INTRODUZIONE}

La medicina trasfusionale è stata oggetto di un processo di profondo rinnovamento e trasformazione, tuttora in rapida evoluzione, che ha visto il passaggio da una fase di semplice raccolta, conservazione e distribuzione del sangue e degli emoderivati a quella di esecuzione di procedure di elevato contenuto tecnologico e significato terapeutico. Risulta, pertanto, importante conoscere i costi di tutte le fasi della lavorazione del sangue per evidenziare i livelli di efficienza del settore e per valutare la congruità del sistema tariffario in vigore rispetto ai costi di esercizio effettivi delle Strutture Trasfusionale (ST). Da tale esigenza è nata l'idea del Progetto CORSA (COsti Raccolta SAngue), condotto con il patrocinio scientifico della Società Italiana di Medicina Trasfusionale e Immunoematologia (SIMTI), con l'obiettivo di stimare il costo medio per tipologia di prestazione su un campione di ST nazionali.

\section{Raccolta e lavorazione del sangue}

In Italia esistono circa 380 ST, ubicate per lo più all'interno delle Aziende Ospedaliere [1].
La legge 10/90, nel definire l'organizzazione del servizio trasfusionale nazionale, individua tre categorie di strutture: Servizi di Immunoematologia e Trasfusione (SIT), Centri Trasfusionali (CT) e Unità di Raccolta (UR). I SIT sono strutture di presidio ospedaliero che operano in bacini di utenza aventi una popolazione di almeno 400.000 abitanti o per un minimo di uno per provincia. I CT sono strutture ospedaliere che possono essere costituite a integrazione dei SIT laddove il bacino di quest'ultimo superi i 400.000 abitanti; essi devono operare in bacini di utenza con una popolazione di almeno 150.000 abitanti. Le UR, infine, sono strutture, fisse o mobili, finalizzate alla raccolta di sangue intero e di plasma; esse dipendono sotto il profilo tecnico e organizzativo dal SIT del territorio di competenza o dal rispettivo CT, laddove sia presente [2]. Le sole altre istituzioni che possono provvedere alla raccolta del sangue sono la Croce Rossa Italiana (CRI) e le associazioni di donatori volontari, fra le quali l'AVIS risulta l'unica di rilievo nazionale.

Le ST possono decidere di raccogliere il sangue al proprio interno oppure di acquistarlo dalle associazioni di donatori sulla base di
* CESAV Centro di Economia Sanitaria Angelo e Angela Valenti dell'Istituto di Ricerche Farmacologiche Mario Negri-Ranica $(B G)$. 
tariffe nazionali stabilite da un apposito decreto legge (D.L. 5/11/1996) [3]. Esistono due tipi di donatori: quelli "abitudinari" (iscritti alle associazioni di volontari) e quelli "occasionali" (non aderendo a nessuna delle suddette associazioni, non garantiscono di fatto periodicità e continuità nelle donazioni).

La trasfusione di sangue può riguardare il sangue intero (dal quale si ottengono con un processo di lavorazione gli emocomponenti, ossia globuli rossi, piastrine e plasma) o la raccolta diretta degli emocomponenti tramite il processo di aferesi.

La separazione degli emocomponenti contenuti nel sangue intero si ottiene utilizzando sacche collegate fra loro in serie da un sistema chiuso, mediante un processo di stratificazione (a seguito di centrifugazione) collegato al diverso peso specifico delle componenti. Tale lavorazione, nonostante il livello elevato di imprecisione, risulta necessaria, in quanto le componenti, oltre ad avere un diverso utilizzo clinico, hanno modalità di conservazione e durata differenti fra loro: le piastrine devono essere separate entro poche ore dal prelievo e conservate, sempre in movimento, per pochi giorni a una temperatura prossima ai $4^{\circ} \mathrm{C}$, mentre i globuli rossi possono essere conservati a $20^{\circ} \mathrm{C}$ per un periodo massimo di circa 40 giorni.

Il processo di aferesi, utilizzando lo stesso principio di una centrifuga che fa precipitare le componenti a peso specifico più alto, permette di estrarre solo l'emocomponente che interessa attraverso attrezzature specifiche, rifondendo direttamente nel donatore quelli non richiesti. Riassumendo, l'attività delle ST può riguardare:

- la raccolta, finalizzata all'ottenimento di unità di sangue intero, piastrinaferesi e plasmaferesi;

- la validazione che include i test diagnostici su tutte le unità di sangue raccolte direttamente dalla ST e di quelle acquistate ancora da validare;

- la separazione del sangue intero raccolto direttamente dalla ST o acquistato, finalizzata all'ottenimento dei concentrati di globuli rossi, piastrine e plasma da separazione.

\section{MATERIALIEMETODI}

Obiettivo dello studio è stato l'analisi dei costi su un campione di ST in Italia, al fine di effettuare stime attendibili sui costi medi delle varie prestazioni erogate. Per il calcolo dei costi medi di struttura sono stati utilizzati i dati forniti dalla contabilità per centri di costo delle strutture coinvolte; quindi, requisito indispen- sabile per la partecipazione allo studio è stata la disponibilità di un sistema analitico di controllo di gestione dell' azienda ospedaliera.

Per poter valutare il campione sotto il profilo dimensionale, le ST sono state ordinate in base al numero di dipendenti, con un totale di 5 addetti per il centro più piccolo e di 60 addetti per quello più grande.

La configurazione di costo adottata è stata quella di costo pieno [4], con imputazione per tipo di prodotto. I prodotti per cui è stato possibile effettuare una stima attendibile del costo medio sono stati:

a. unità di plasma da aferesi;

b. unità di piastrine da aferesi;

c. unità di sangue intero.

Sono state, inoltre, individuate sei macrocategorie di costi per cui è stato definito un idoneo criterio di imputazione.

1. Il personale comprende il costo di tutto il personale impiegato all'interno della ST per la raccolta e la lavorazione del sangue (medici, infermieri, biologi, tecnici, amministrativi e ausiliari), stimato in base ai carichi di lavoro dichiarati dai centri stessi in appositi questionari.

2. Il materiale diagnostico comprende tutte quelle voci che sono afferenti alla diagnostica (tipicamente i reagenti).

3. La categoria definita come altro materiale sanitario considera tutto il materiale sanitario non diagnostico consumato dalla ST (sacche per la raccolta di sangue intero, kit per aferesi ecc.), unitamente ai farmaci eventualmente somministrati ai donatori nel corso della donazione stessa.

4. Il materiale non sanitario comprende tutte le voci di costi non sanitari (ad esempio, la cancelleria e il restante materiale economale).

5. La voce attrezzature riguarda i costi di manutenzione e riparazione delle apparecchiature esistenti, unitamente agli ammortamenti contabilizzati per quelle di proprietà della ST.

6. I costi generali comprendono la quota di spese generali per cui non è possibile una imputazione diretta (amministrazione, lavanderia, pulizia, utenze generali, ecc.).

Per poter attribuire a ciascun prodotto $\mathrm{i}$ costi specificamente afferenti, l'attività svolta dalla ST è stata suddivisa in quattro categorie: raccolta, validazione, separazione, altro. Alla prima sono stati imputati tutti i costi afferenti alla mera attività di raccolta del sangue operata direttamente dalla ST e finalizzata all'ottenimento di unità di sangue intero, 
piastrinaferesi e plasmaferesi. La validazione comprende i costi afferenti l'esecuzione dei test diagnostici seguiti per tutte le unità di output che lo richiedono. Alla terza categoria sono imputati i costi sostenuti per la separazione del sangue intero, finalizzata principalmente all'ottenimento dei concentrati di globuli rossi, piastrine e plasma da separazione. Infine, l'ultima voce riguarda i costi generali attribuibili in ugual misura a tutte le unità di emocomponenti che transitano dalla ST.

La figura 1 riassume lo schema organizzativo adottato dalle ST nella lavorazione del sangue, mentre la figura 2 mostra lo schema di imputazione dei centri nelle varie attività delle ST.

Il lavoro è stato suddiviso organizzativamente in tre diverse fasi, ciascuna finalizzata all'individuazione delle ST in grado di fornire le informazioni di carattere contabile e gestionale necessarie per il raggiungimento dell'obiettivo finale.

\section{Fase 1}

Al fine di poter stimare il costo medio per tipologia di prestazione su un campione il più ampio e esaustivo possibile, è stata concessa l'opportunità a tutte le strutture aderenti alla SIMTI di partecipare in modo spontaneo e volontario alla prima fase del progetto. Il questionario anagrafico è stato, infatti, inviato a tutte le ST presenti sul territorio italiano, evidenziando la necessità di disporre di un report di controllo di gestione come condizione indispensabile per una partecipazione completa allo studio. Dei 380 questionari informativi inviati, ne sono pervenuti compilati 116, di cui 76 hanno dichiarato di essere dotati dei requisiti di carattere contabile necessari ai fini dello studio.

\section{Fase 2}

$\mathrm{Si}$ è quindi proceduto nello studio inviando un ulteriore questionario alle 76 ST, selezionate nella prima fase, ritenute idonee ad accedere alla seconda fase del progetto.

Il secondo questionario ha permesso di conoscere con un grado di dettaglio maggiore la struttura organizzativa delle ST rispetto ai servizi prestati, unitamente all'attività svolta dalle stesse. Sono state raccolte informazioni di carattere organizzativo/gestionale, attraverso la formulazione di domande specifiche volte a comprendere la produttività totale annua della struttura suddivisa per tipologia di prodotto, la destinazione di tutte le unità prodotte, la dotazione di apparecchiature della struttura e il personale ivi impiegato, con la stima dei carichi di lavoro nelle diverse attività svolte. Questa seconda analisi ha portato a un'ulteriore selezione. Sono, infatti, pervenuti com- pilati 45 questionari e, fra questi, solamente 30 contenenti delle risposte complete ed esaurienti, necessarie per partecipare alla terza fase dello studio focalizzata sui costi veri e propri. L'elaborazione delle informazioni organizzative contenute nel secondo questionario è stata oggetto di una pubblicazione a se stante [5].

\section{Fase 3}

L'ultima fase della ricerca ha riguardato l'analisi dei report della contabilità per centri di costo, richiesti alle $30 \mathrm{ST}$ così selezionate, al fine di individuare uno schema di analisi co-

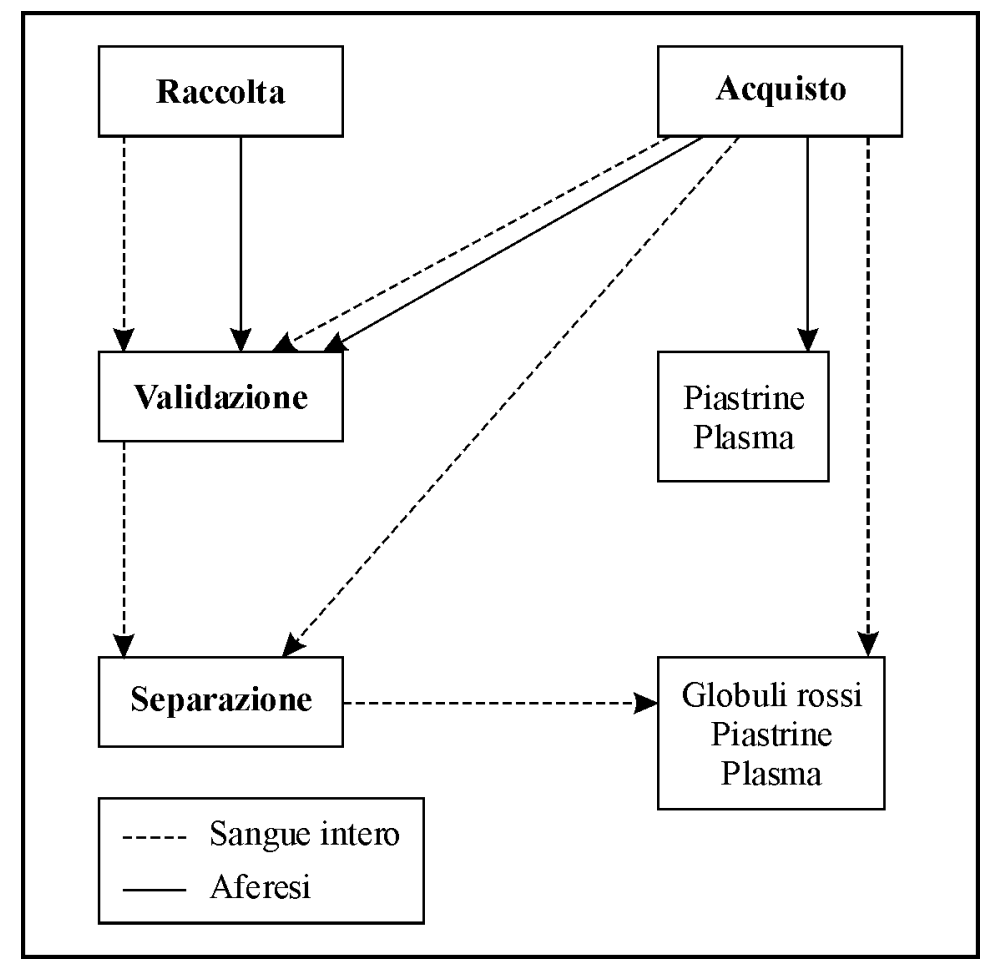

Figura 1

Classificazione funzionale dell'attività svolta dai centri trasfusionali

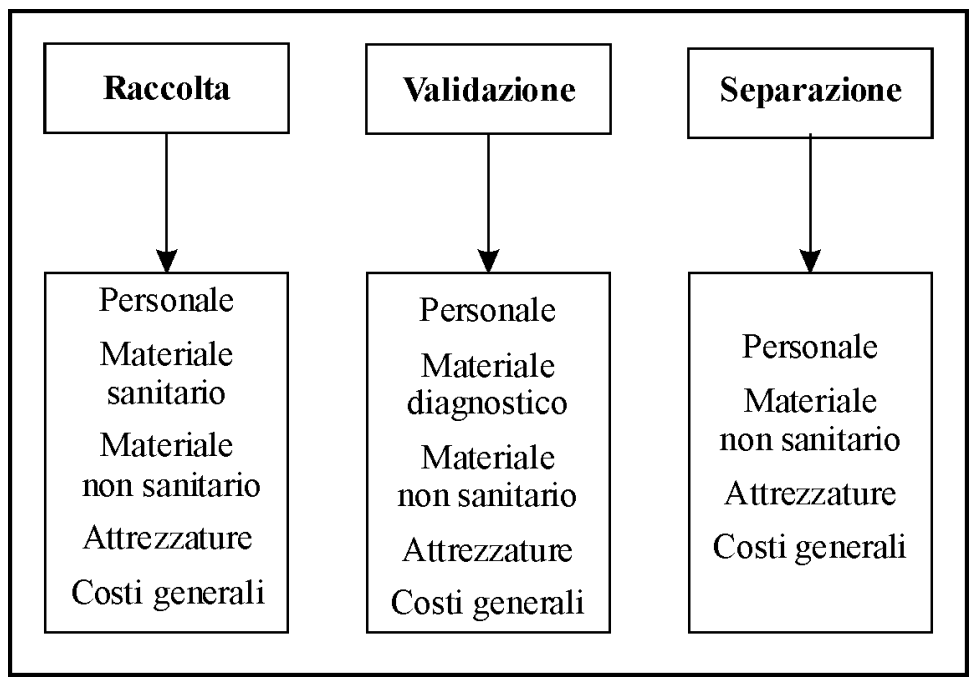

Figura 2

Imputazione dei costi in base alle attività individuate 
mune a tutte le strutture coinvolte. Solamente 10 ST hanno risposto in modo esauriente, dimostrando di possedere una reportistica sufficientemente dettagliata per consentire le elaborazioni necessarie a stimare il costo medio per tipologia di prestazione. La drastica riduzione della numerosità del campione finale rispetto a quello iniziale è sembrata solo in minima parte riconducibile alla scarsa collaborazione degli operatori coinvolti, ma piuttosto ai limiti oggettivi tuttora esistenti nel sistema di controllo di gestione di ASL e aziende ospedaliere.

\section{RISULTATI}

L'analisi dei costi dei tre prodotti (plasmaferesi, piastrinaferesi, sangue intero) è riportata in Tabella 1. Nei dieci centri presi in esame la piastrinaferesi è risultata il prodotto più costoso, con un costo medio di euro 285,8 ;

\begin{tabular}{|c|c|c|c|c|}
\hline Prodotto & & $\begin{array}{l}\text { Plas maferesi } \\
\text { (Euro) }\end{array}$ & $\begin{array}{c}\text { Piastrinaferesi } \\
\text { (Euro) }\end{array}$ & $\begin{array}{c}\text { Sangue intero } \\
\text { (Euro) }\end{array}$ \\
\hline \multirow{10}{*}{ Centro } & A & 107,8 & - & 79,7 \\
\hline & B & 177,3 & 525,9 & 108,0 \\
\hline & $\mathrm{C}$ & 254,8 & 636,3 & 209,2 \\
\hline & $\mathrm{D}$ & 123,3 & - & 111,5 \\
\hline & $\mathrm{E}$ & 98,7 & 109,8 & 79,1 \\
\hline & $\mathrm{F}$ & 230,2 & 305,7 & 199,7 \\
\hline & G & 145,0 & 553,9 & 92,4 \\
\hline & $\mathrm{H}$ & 180,6 & 273,2 & 177,2 \\
\hline & I & 111,7 & 453,2 & 104,3 \\
\hline & $\mathrm{L}$ & 109,2 & - & 130,3 \\
\hline Totale & & 153,9 & 285,8 & 129,1 \\
\hline $\mathrm{IC} *$ & & $114,5-193,2$ & $107,8-463,8$ & $94.3-164$ \\
\hline
\end{tabular}

Tabella 1

bella ultima riga

\section{Figura 3}

Composizione del costo totale in ogni centro la plasmaferesi ha un costo minore, pari a euro 153,9; il sangue intero è il prodotto con il costo inferiore, pari a euro 129,1. Per stimare il costo degli emocomponenti da separazione, si è suddiviso ulteriormente il costo della sacca di sangue intero raccolto, utilizzando i valori delle tariffe nazionali stabilite nel decreto 16/12/1996 come base di ponderazione. I costi così stimati sono, rispettivamente, di euro 17,7 per unità di globuli rossi da separazione, euro 21,6 per plasma da separazione e euro 89,8 per piastrine da separazione.

Gli intervalli di confidenza sia della plasmaferesi che del sangue intero indicano una variazione di quasi il 100\%. Infatti, per la plasmaferesi tale intervallo varia tra euro 114,5 e euro 193,2, mentre per il sangue intero varia tra euro 94,3 e euro 164,0. Per quanto riguarda la piastrinaferesi la variazione del valore medio è di gran lunga più ampia (400\%), con un corrispondente intervallo di confidenza compreso tra euro 107,8 e euro 463,8 .

Si è cercato di ricavare qualche indicazione interessante in relazione alla dimensione produttiva delle ST, assunta pari al numero di unità di emoderivati per anno, calcolando coefficienti di correlazione parziale fra dimensione della ST e incidenza di ciascuna voce di costo (costo del personale, costo del materiale diagnostico, sanitario e non sanitario, attrezzature e costi generali) (figura 3 ) per i tre prodotti oggetto dell' analisi.

Solamente per la plasmaferesi è stata trovata un' associazione parziale negativa fra volume di produzione e costo del personale sanitario $(\mathrm{r}=-0,9594, \mathrm{p}=0,01)$ e del materiale sanitario $(\mathrm{r}=-0,9532, \mathrm{p}=0,012)$. La relazione è invece positiva per il costo dell' attrezzatura $(\mathrm{r}=0,9468$, $\mathrm{p}=0,015)$. Per le rimanenti voci di costo, così come per la piastrinaferesi e il sangue intero, le correlazioni sono risultate statisticamente non significative.

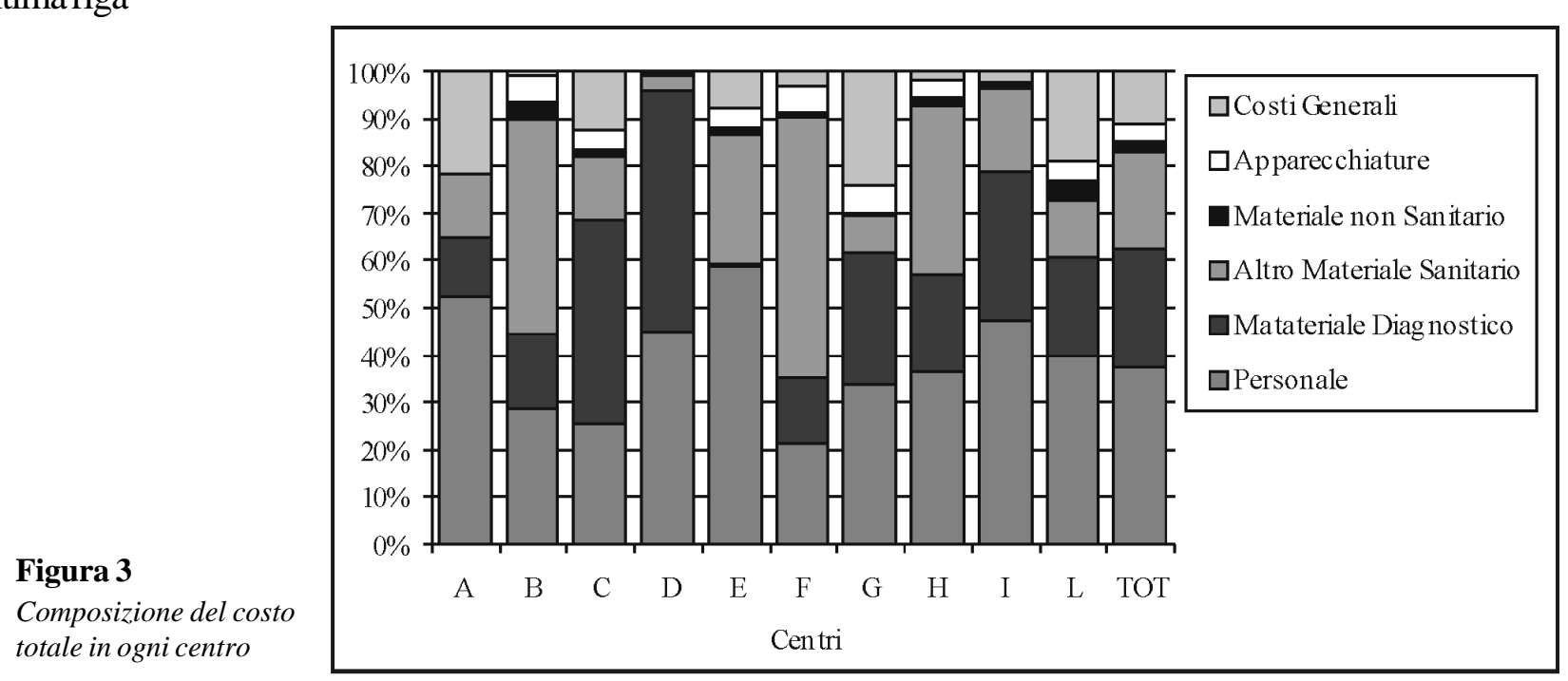




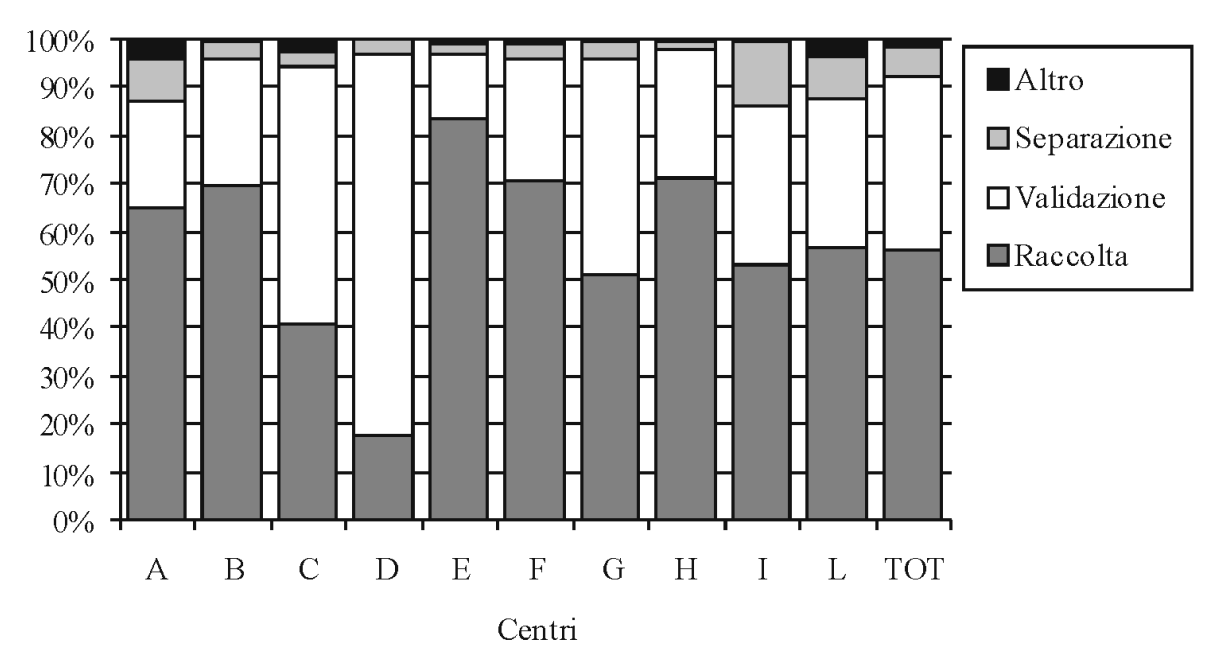

Figura 4

Incidenza del costo delle varie aree $d i$ attività
Dall' analisi dei dati, è emersa una maggior propensione dei centri più piccoli alla raccolta di sangue intero, mentre i centri medio-grandi sembrerebbero dedicare una quota maggiore delle attività all' aferesi. Per testare questa ipotesi, sono stati calcolati i coefficienti di correlazione parziale fra la dimensione della ST e la composizione dei costi totali disaggregati in funzione delle tre tipologie di attività, assunta pari alla proporzione dei costi attribuita alle tre aree funzionali del processo di lavorazione di emoderivati (figura 4). La relazione fra volume totale della produzione e proporzione delle risorse dedicate alla raccolta $(\mathrm{r}=-0,07)$, alla validazione $(\mathrm{r}=0,02)$ e alla separazione $(\mathrm{r}=0,248)$ non è risultata statisticamente significativa. Pertanto, i dati raccolti non supportano l'ipotesi di economie di scala o di specializzazione nella produzione di prodotti emoderivati.

\section{DISCUSSIONE}

Il Progetto CORSA ha permesso di stimare un costo medio per prodotti di alcuni processi produttivi delle ST, attraverso l' analisi dei dati provenienti dal sistema di controllo di gestione del campione selezionato. Risulta importante sottolineare che l'analisi dei costi del processo di raccolta e lavorazione del sangue eseguito dalle ST ha portato all'individuazione del costo della sola attività svolta direttamente dalla struttura. Non sono, quindi, incluse le tariffe pagate per l'acquisizione di unità raccolte da altre strutture, né i contributi versati alle associazioni di donatori, in quanto frutto di transazioni economiche fra soggetti diversi (e non di costi generati dai processi produttivi delle ST).

Per pervenire alla stima di un costo medio per prestazione all'interno di ciascuna struttu- ra, le voci di costo provenienti dal report di controllo di gestione sono state incrociate con i dati organizzativi raccolti nei due questionari. Solamente il $13.3 \%$ delle ST inizialmente interpellate è stato in grado di fornire dati disaggregati a livello di servizio, necessari per giungere alla stima del costo medio per tipologia di prestazione. Tale risultato evidenzia come il processo di aziendalizzazione delle ASL e delle Aziende Ospedaliere (sancito legislativamente dai Dlgs 502/92, 517/93 e successivi aggiornamenti), mirato a contribuire a un' allocazione efficiente delle risorse nell' ambito delle singole strutture, sia ancora lontano da una completa attuazione in materia di riorganizzazione della struttura contabile.

Sembrerebbe particolarmente utile affinare il sistema di contabilità analitica all'interno delle varie ST per misurare e confrontare il livello di risorse assorbite da parte dei singoli processi, vista la notevole differenza riscontrata fra $i$ costi delle diverse prestazioni effettuate in un processo produttivo apparentemente molto standardizzato quale quello del sangue.

Il limite principale di questo studio è, quindi, la numerosità ridotta del campione finale che non ha permesso di ottenere stime molto solide, come evidenziato anche dagli ampi intervalli di confidenza delle stime stesse. Pur consapevoli di questo limite, il risultato principale di questo studio sembra la quasi totale assenza di correlazioni fra la dimensione delle ST e i costi dei processi produttivi del sangue.

\section{Ringraziamenti}

Si ringrazia la Dott.ssa Cecilia Viscarra e il Dottor Graziano Schiavone per l'utile apporto sia medico che statistico nella stesura dell'articolo. 
Il costo medio per tipologia di prestazione nei centri trasfusionali

\section{BIBLIOGRAFIA}

1. Piano nazionale sangue e plasma 1999-2001. Il Sole 24 Ore Sanità; 7-13 Dicembre 1999.

2. Istituto Superiore di Sanità. Mappa delle strutture trasfusionali esistenti sul territorio nazionale; 1995.

3. Decreto 5 Novembre 1996. Gazzetta Ufficiale della Repubblica Italiana n. 294 del 16 Dicembre 1996.

4. Anthony RN, Young DW. Controllo di gestione per gli Enti Pubblici e le Organizzazioni Non Profit. Mac Graw Hill, Milano, 1992.

5. Cornago D, Garattini L. Efficienza tecnologica e produttiva delle strutture trasfusionali in Italia. Farmeconomia e percorsi terapeutici 2002; 3(1): 28-35. 